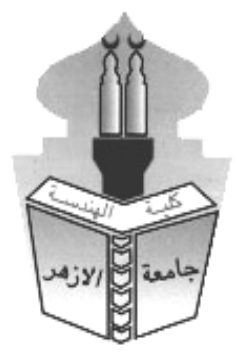

\title{
ASSESSMENT OF DTMS GENERATED USING DIFFERENT WAVELENGTHS OF MULTISPECTRAL LIDAR IN COASTAL AND FORESTED AREAS
}

\author{
A. Mandouh*1, Lamyaa Gamal El-deen Taha ${ }^{1}$, Mahmoud El Nokrashy O. Ali ${ }^{2}$, and \\ Mostafa H. A. Mohamed ${ }^{2}$ \\ ${ }^{1}$ Department of Aviation and Aerial Photography, National Authority for Remote Sensing and \\ Space Sciences, Cairo, Egypt. \\ ${ }^{2}$ Department of Civil Engineering, Faculty of Engineering, Al-Azhar University, Cairo, Egypt \\ *Corresponding Author E-mail: asmaa.ahmed@narss.sci.eg
}

\begin{abstract}
The penetration ability of the Green wavelength in multispectral Light Detection and Ranging (LiDAR) pulses into water bodies makes it a valuable tool in DTM generation for land and certainly bathymetry. Titan is the first active multispectral LiDAR that extends information for 3D point clouds from a single sensor. It is provided by Teledyne Optech is an example of the multispectral LiDAR system. It has three different wavelengths mid-infrared (MIR), nearinfrared (NIR), and green $(1.550,1.064$, and $0.532 \mu \mathrm{m})$ respectively. This research aims to compare the performance of two ground-filtering algorithms to obtain digital terrain models in the forest and coastal areas. The tested area is located at the Southwest tip of Tobermory (Ontario, Canada). Two filtering algorithms are used; Adaptive Triangulated Irregular Network (ATIN) and Elevation Threshold with an Expand Window (ETEW). The two filtering algorithms performance were evaluated using the cloud to cloud (C2C) method provided by cloud compare software. The results show that ATIN gives results better than ETEW in the three different wavelengths of multispectral LiDAR data.
\end{abstract}

KEYWORDS: DTM, Multispectral LiDAR, Filters, Open Source Software, and Point Cloud.

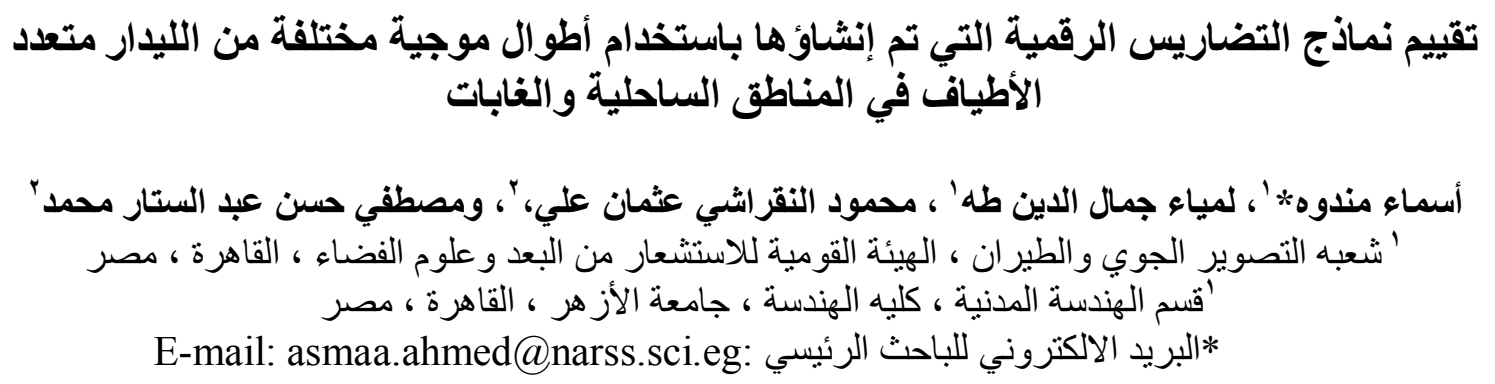

إن قدرة اختراق الطول الموجي الأخضر في نبضات الضو ه متعدد الأطياف والمدى (الليدار ) في الأجسام المائية تجعله أداة

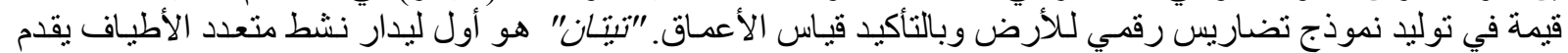

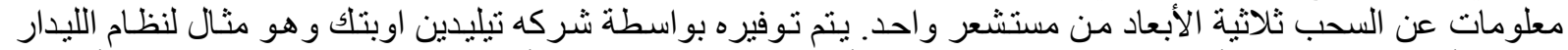

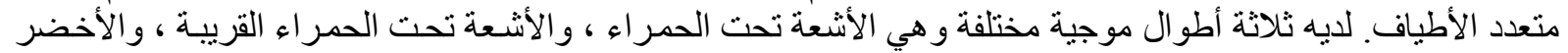




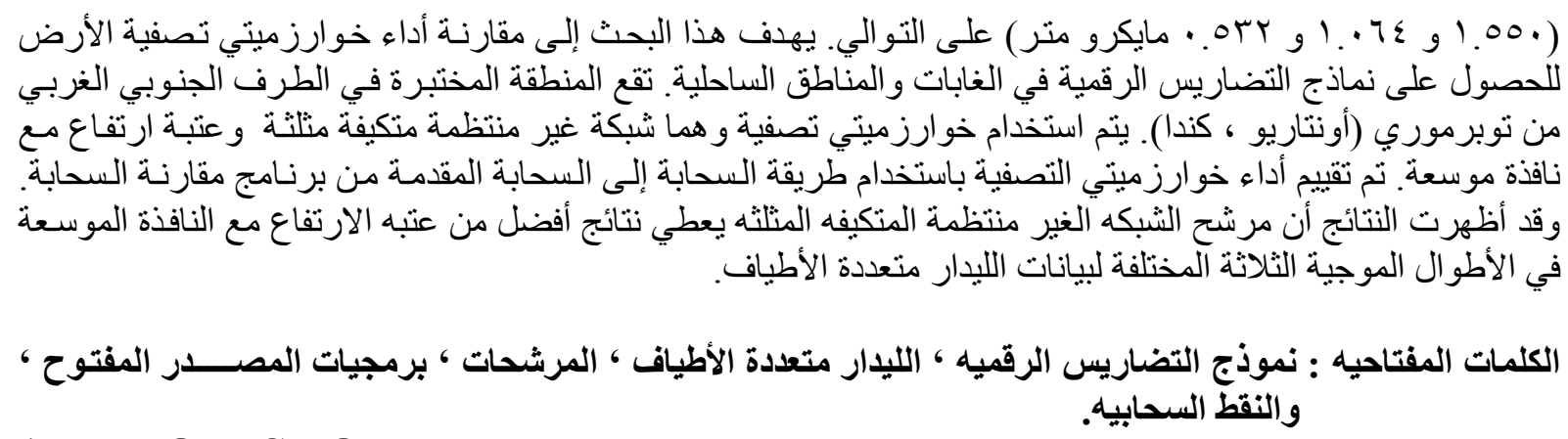

\section{INTRODUCTION}

Multispectral Airborne Laser Scanning (MALS) has become one of the primary remotesensing technologies for analyzing the land and water surface due to its good capability of penetrating the water surface and three-dimensional information acquisition (Chen, 2018). Multispectral LiDAR is a gauging technique that surveys distance to an object, which can record a set of points that describe the target object. Compared with mono-wavelength LiDAR; multispectral LiDAR data have the advantage of acquiring more accurate bathymetry information in the water bodies.

Digital Terrain Models (DTMs) derived from LiDAR are widely used as important geospatial information sources for various remote sensing applications, including the precise orthorectification of high-resolution satellite images, 3D spatial analyses, multi-criteria decision support systems, and deformation monitoring (Alganci et al., 2018). The accurate and successful DTM is essential to ensure an accurate representation of the ground and any feature above the ground; so, it is important to select the most appropriate ground filtering algorithm by considering the aim, accuracy requirement, and environment of the study area (Alganci et al., 2018).

Actually, there are many ground algorithms available for filtering ground and a non-ground point cloud from airborne LiDAR data; some of them can be found in ALDPAT software; open-source software provided by International Hurricane Research Center, Department of Environmental Studies from Florida International University, USA (Zhang and Cui 2007). The most well-known algorithms are those Adaptive TIN (ATIN) (Axelsson, 2000), Elevation Threshold with Expand Window (ETEW) (Zhang et al., 2003); Progressive Morphology (Morph) (Zhang and Whitman 2005), Maximum Local Slope (Slope) (Vosselman, 2000).

Although there many of studies have addressed the performance of the multispectral LiDAR for land water and land cover classification such as (Morsy et al., 2018; Morsy et al., 2017; Chen, 2018 and Shaker et al., 2019) and also several previous studies to performance algorithms for filtering ground returns from airborne LiDAR datasets, especially in planted forest environments. Sulaiman et al., (2010) used a set of ground filtering algorithms provided by ALDPAT and its performance was evaluated by a commercial program Terracan. These algorithms were tested on a test area involved forestry area and just a little neighborhood area. The ETEW filter has found the closest surface to the surface produced by TerraScan with $47 \mathrm{~mm}$ standard deviation. He suggested to use the Adaptive TIN in urban areas since it can handle unstable surfaces. Serifoglu et al., (2016) examined ground filtering algorithms using the point cloud extracted from the aerial images taken from a UAV and showed that the ATIN algorithm is the most successful one, while the ETEW algorithm was found as the least successful ones. It also concluded that the higher of point density, the performance of the filtering algorithms decreased. Considering this theme, the main objective of this study was to extract DTMs using multispectral LiDAR data in coastal and forest environments. This is by using two ground-filtering algorithms ATIN and ETEW and comparing the performance of them using the cloud to cloud (C2C) distance calculation method provided by cloud compare software, this is the simplest and fastest direct 3D comparison between point clouds (Lague et al.,2013).

Cloud Compare is a 3D point cloud processing program provided by Daniel Girardeau. It was originally designed to compare two-point clouds of three-dimensional (Daniel G. 2015). Also, 
can compare a point cloud with a 3D mesh. It adop ts an octree structure that is highly optimized for dealing with the number of points more than 10 million points.

\section{MULTISPECTRAL LIDAR SYSTEM}

The first operational multispectral airborne laser scanners system was launched by Teledyne Optech, Ontario, Canada in late 2014 with the product name Titan. With this scanner, active multispectral information is for the first time available for 3D ALS point clouds from a single sensor. The channels of the sensor are infrared at $1.550 \mu \mathrm{m}$ (Channel-1), near-infrared 1.064 $\mu \mathrm{m}$ (Channel-2) and green $0.532 \mu \mathrm{m}$ (Channel-3). Each of the channels produces a separate point cloud (Matikainen et al., 2017). This gives access to a variety of information about an area with different spectral properties of the objects. Table (1) shows the characteristic of Titan multispectral LiDAR and figure (1) shows the components of Titan multispectral lidar system which consist of a flight management system, an operator laptop, a digital camera, a laser scanner assembly, a Global Navigation Satellite System (GNSS), an Inertial Measurement Unit (IMU), and a control and data recording unit are fundamental parts of a multispectral lidar system (Ma et al., 2019).

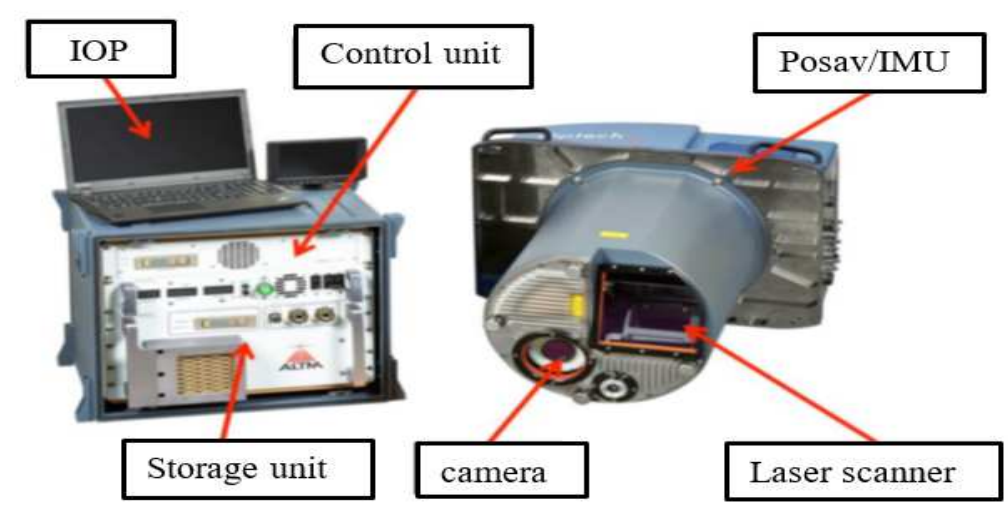

Figure (1): Teledyne Optech Titan system (Optech T., 2015).

Table (1): the characteristics of Optech Titan multispectral LiDAR(Chen, 2018).

\begin{tabular}{|l|l|}
\hline Parameter & Specification \\
\hline Wavelengths & $\begin{array}{l}\text { Channel 1: } 1550 \mathrm{~nm} \text { (shortwave infrared, SWIR) } \\
\text { Channel 2: } 1064 \mathrm{~nm} \text { (near-infrared, NIR) } \\
\text { Channel 3: } 532 \mathrm{~nm} \text { (green, G) }\end{array}$ \\
\hline Forward Angles & $\begin{array}{l}\text { Channel } 1: 3.5^{\circ} \\
\text { Channel 2: } 0^{\circ} \\
\text { Channel }: 7^{\circ}\end{array}$ \\
\hline Pulse repetition frequency (PRF) & $\begin{array}{l}\text { Programmable; } 50-300 \mathrm{kHz} \text { per channel; } 900 \mathrm{kHz} \text { in } \\
\text { total }\end{array}$ \\
\hline Scan Frequency & Programmable; $0-210 \mathrm{~Hz}$ \\
\hline Point density & $\begin{array}{l}\text { Bathymetric: }>15 \mathrm{pts} / \mathrm{m} 2 \\
\text { Topographic: }>45 \mathrm{pts} / \mathrm{m} 2\end{array}$ \\
\hline Accuracy & $\begin{array}{l}\text { Horizontal: } 1 / 7,500 \times \text { altitude, } 1 \sigma \\
\text { Vertical: }<5-10 \mathrm{~cm}, 1 \sigma\end{array}$ \\
\hline Laser range precision & $5<0.008 \mathrm{~m} ; 1 \sigma$ \\
\hline
\end{tabular}




\section{DATA SOURCES}

The testing data used in the experiments obtained from ISPRS Commission III on 'Remote Sensing'-and WG 111/5. As shown in figure (2) this data covered a natural coastal area located in Tobermory, Ontario, Canada. For the experimental testing, A $1.5 \mathrm{~km}$ by $1 \mathrm{~km}$ subset of multispectral LiDAR data was clipped. The main terrain of this test area is forest and coastal areas, in addition to a few residential buildings. The total number of points for each dataset is over 24 million for channel-1 and channel-2, but channel-3 has over 34 million points.

Flight parameters used in the Multispectral Laser Scanning campaign:

- Wavelengths: $1550 \mathrm{~nm}$ (ch-1), $1064 \mathrm{~nm}$ (ch-2), and $532 \mathrm{~nm}$ (ch-3).

- Field of view: 40 degrees.

- Flight height: $457 \mathrm{~m}$.

- Flight speed: $72 \mathrm{~m} / \mathrm{s}$.

- Laser Pulse Repetition Frequency (PRF): $625 \mathrm{kHz}$ in total.

- The number of ALS Strips: 11 for each wavelength.

- Average point density: 12.99, 13.19, and 18.58 points $/ \mathrm{m} 2$ for channel-1, 2, and 3 respectively.

- The number of returns: 4.

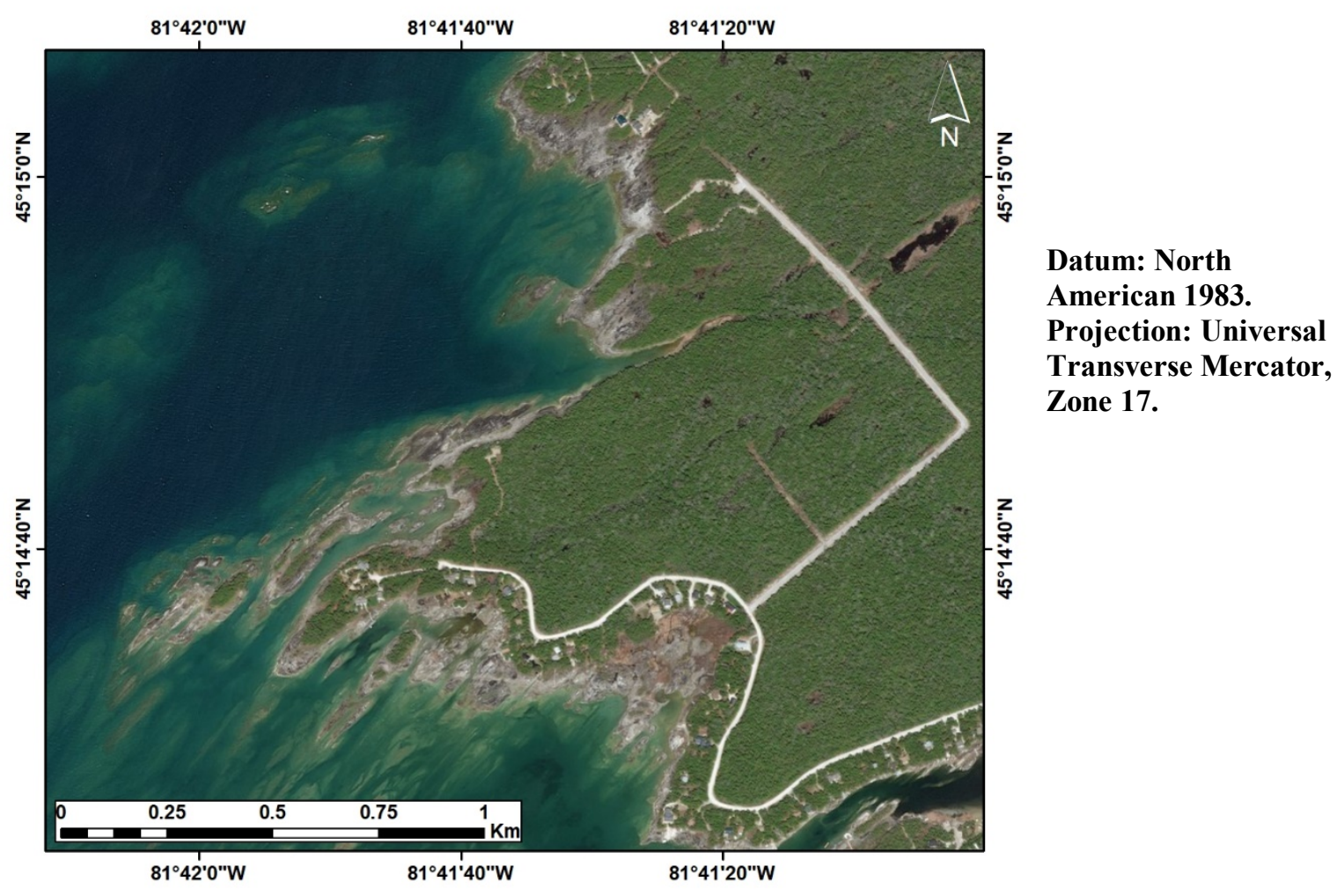

Figure (2): Tested area.

\section{METHODOLOGY}

Optech Titan multispectral LiDAR data was used in this paper to generate DTMs with the three different wavelengths using two filtering algorithms ATIN and ETEW provided by ALDPAT v.1.0 open-source software. This software was developed by the national center for airborne laser mapping (Zhang and Cui, 2007). Two DTMs are produced from each wavelength separately. The method consists of three elementary steps:

The first step, preparing multispectral lidar data by merged all 11 strips of each channel in one strip and segmented the test areas. This step was done by using Cloud Compare software recommended by (Dai et al., 2018). 
In the second step, ground filtering by using ATIN and ETEW ground algorithm filters from ALDPAT software, we generate DTMs from channel-1, 2 and 3.

The third step, the evaluation of the performance of two selected filtering algorithms in the three different wavelengths. In this step, the focus was on quantitative assessment. A wellknown commercial software ENVI-LiDAR was chosen to produce a high-precision digital terrain model and use it as a reference to evaluate the performance of the two filtering algorithms with the three different wavelengths.

\subsection{Pre-processing Multispectral LiDAR Data}

First, the data has been pre-processed by Optech, as the company has calibrated all points by geoid correcting and aligned the three channels automatically with a program of Optech's LiDAR Mapping Suite. The data pre-processing stage includes several tasks: the cleaning, merging data and cutting three-dimensional cloud data using a set of algorithms provided by cloud compare.

CH-1 $(1550 \mathrm{~nm})$
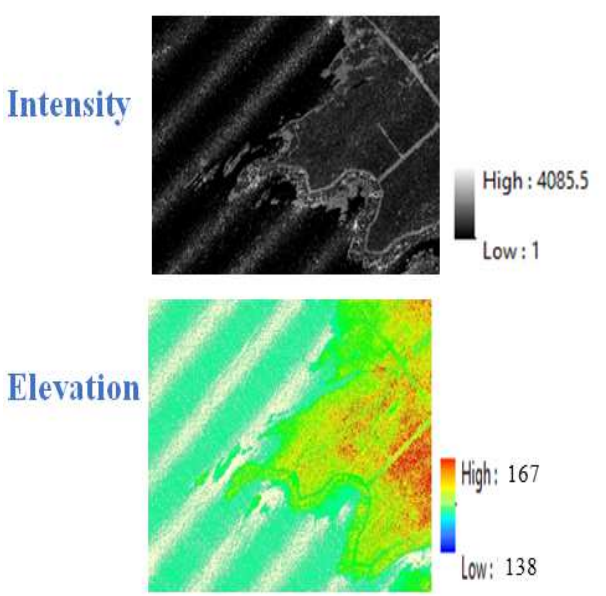

CH-2 (1064 nm)
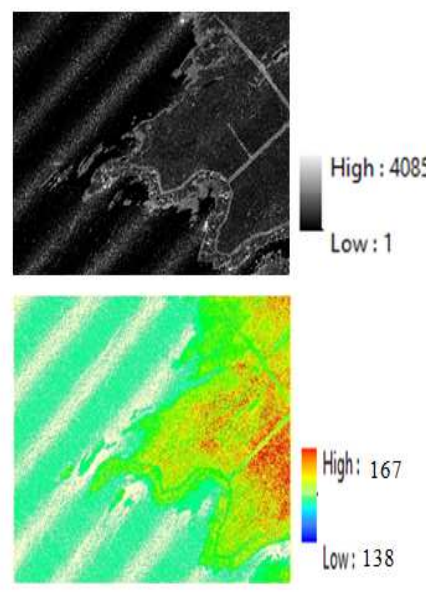

CH-3 (532 nm)

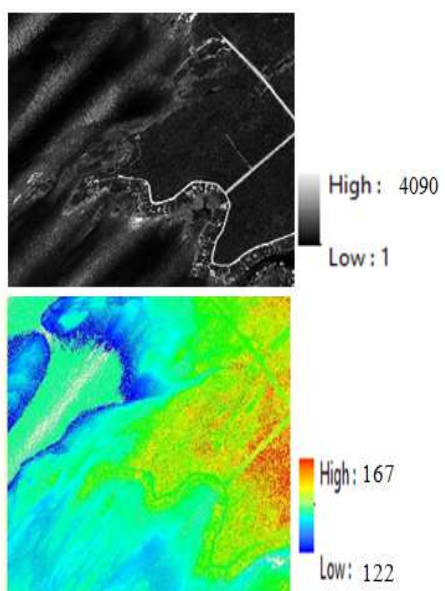

Figure (3): Different intensity, elevation, and height above ground for the three LiDAR channels.

Figure (3) shows the clear differences between the three channels according to intensity and elevation. As the reflection rate varies according to the surface and the target material, the intensity information of the points from vegetation, bare-earth, or water surface is different. In terms of intensity; the water area in ch-3 has high-intensity values compared with other channels. The intensity value in $\mathrm{CH}-1$ and $\mathrm{CH}-2$ is very close in the tree and water areas. In terms of elevation; the elevation of $\mathrm{CH}-1$ and $\mathrm{CH}-2$ is very close; while the difference appeared in channel-3. The lowest elevation of channel-3 is lower than the lowest elevation of $\mathrm{CH}-1$ and $\mathrm{CH}-2$ by $16 \mathrm{~m}$. This is due to the ability of the green wavelength to penetrate the surface of the water and recorded points of the benthic water layer.

\subsection{Ground Filtering Algorithms}

The data filtering stage comes to classify the point clouds into ground points and non-ground points. In this study, the Adaptive TIN (ATIN) and Elevation Threshold with Expand Window (ETEW) algorithms were used to filter the point clouds extracted from the multispectral airborne LiDAR. All these algorithms were executed in the Airborne LiDAR Data Processing and Analysis Tools (ALDPAT) v.1.0 software. The ALDPAT software is developed by the International Hurricane Research Center, Department of Environmental Studies, Florida International University, USA (Zhang and Cui, 2007). Each algorithm has its specification is shown in table (2) and the capability of every filtering algorithm in ALDPAT depends on the topography of the data itself. 


\subsubsection{Adaptive Triangulated Irregular Network (ATIN) Filter}

In this filtering algorithm, the area is subdivided into cells and taking into consideration that the cell size should be extended the size of a non-ground biggest object. Then selected the lowest height points in each cell and considered these local minimum points as ground points. Next, a rough TIN is generated based on seed points using the Delaunay triangulation algorithm. All the points except for seed points are examined in terms of their distance to the TIN surface and a maximum of three angles between the triangle surfaces. If the distance and angle of a point are less than the predefined threshold, the point would be added to the ground points. The TIN is created using these new points. This process iteratively continues until all points are classified as ground or non-ground (Zhang and Cui, 2007; Suleymanoglu and Soycan, 2019).

\subsubsection{Elevation Threshold with an Expand Window (ETEW) Filter}

This filtering algorithm a set of square cells, and all points, except the minimum height, are ignored. For the next occurrence, the cell size is increased and the minimum height is specified in each cell. After that, all points with elevations above the minimum are rejected. The process is repeated as cells and thresholds increase in size until there is no point to remove in the previous iteration. Formulas are supported by (Zhang and Whitman 2005) ;(Zhang and Cui 2007).

$$
\begin{gathered}
Z_{t, j}-Z_{i, \min }>h_{i, \tau} \\
h_{i, \pi}=\mathrm{S} * C_{i} \\
C_{i}=2 * C_{t-1} \quad \mathrm{i}=2,3, \ldots \mathrm{N}
\end{gathered}
$$

Table 2: chosen filters and parameters (units per meter).

\begin{tabular}{|l|l|l|}
\hline Algorithm & Adaptive TIN & Elevation threshold with expanded window \\
& ATIN & ETEW \\
\hline Parameters & Cell size: $1.0 \mathrm{~m}$ & Width: $1.0 \mathrm{~m}$ \\
& Z threshold: $0.20 \mathrm{~m}$ & Height: $1.0 \mathrm{~m}$ \\
& Inti. Grid size: $100.0 \mathrm{~m}$ & Z factor: $0.9 \mathrm{~m}$ \\
& Tile X length: 200 & Slop: 1.0 \\
& Tile Y length $: 200$ & Loop times: 10 \\
\hline
\end{tabular}

\subsubsection{Filtering Process in ENVI-LiDAR}

In order to complete the comparison process and evaluate the performance of the two available ground filters in an open-source program, the same data were also processed using the well-known commercial software ENVI- LIDAR version 5.3.0, available it National Authority for Remote Sensing and space sciences (NARSS). It is a useful version for filtering LiDAR point clouds into the ground and non-ground points and has the ability to convert georeferenced LiDAR points into layers of GIS and can then be exported to many output formats, $3 \mathrm{D}$ visual databases, orthophoto, digital surface models (DSM), digital elevation models (DEMs), digital elevation model contours, terrain TIN, contour lines and construction (site, Vector ocean, ceiling face vectors), trees (location, height, height, radius), power lines (power line vectors, power pole list, power line attachment point list) (Harris Corporation, 2019). According to (Badenko et al., 2019) this software is very useful for realistic visualization. It allows us to quickly and qualitatively classify buildings, power-lines, and high vegetation. The ENVI-LiDAR API leverages the power of IDL to perform specialized tasks and add custom functionality to ENVI-LiDAR. The ENVI-LiDAR can be used to extend toolbox extensions with ENVI LiDAR, batch process, and write ENVI-LiDAR route lines (Harris Corporation, 2019).

\subsection{Quantitative Assessment}

The quantitative analysis was done by using the cloud to cloud $(\mathrm{C} 2 \mathrm{C})$ absolute distance by cloud compare software. Cloud Compare is a $3 \mathrm{D}$ point cloud processing program provided by 
Daniel Girardeau. It was originally designed to compare two-point clouds of threedimensional (Daniel G. 2015). Also, it can compare a point cloud with a 3D mesh. It adopts an octree structure that is highly optimized for this use case. This is because the number of points reaches more than 10 million points with 2 GB of memory.

In this research, the 3D surface deviation analysis is carried out using the $\mathrm{C} 2 \mathrm{C}$ distance computation method via cloud compare software. The first step is the determination and the selection of the reference and the compared datasets. The reference dataset is the DTM from Envi-LiDAR and the other dataset is the ALDPAT DTMs as shown in figure (4a). Figure (4b) shows that major account parameters provided by cloud compare, including split $\mathrm{X}, \mathrm{Y}$, and Z components which can generate 3 additional numerical fields that correspond to the distance (absolute) between each comparison point and its closest reference point along each dimension (i.e. this corresponds to 3 components of the deviation vector).

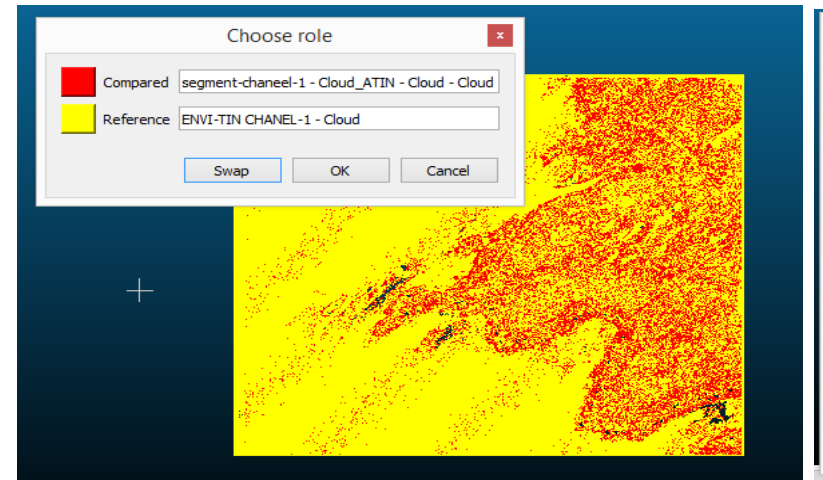

(a)

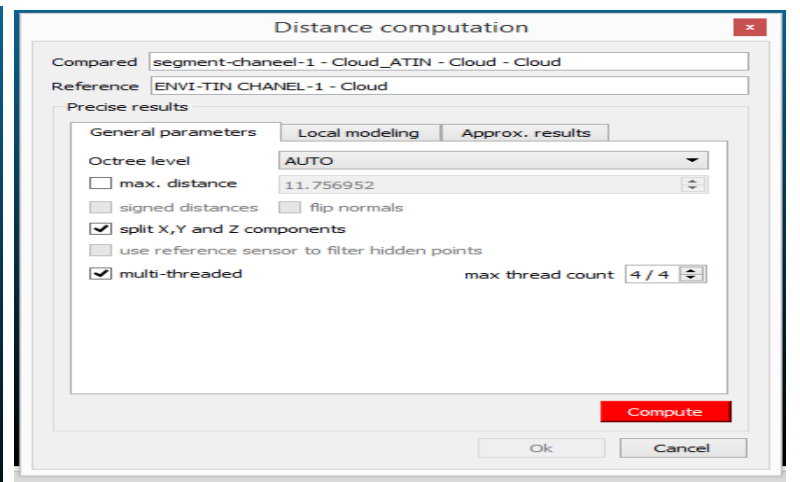

(b)

Figure (4): The selection of datasets to be used in the $\mathrm{C} 2 \mathrm{C}$ distance computation process: a) define the roles of each cloud, b) the main parameters for the computation process.

The $\mathrm{C} 2 \mathrm{C}$ distance calculation method provided by cloud compare software is the simplest and fastest direct 3D comparison between point clouds because it does not require converting point clouds to grids or meshing data, nor calculating surface normal values (Lague et al.,2013). For each point in the compared cloud, C2C searches to find its closest point in a reference cloud and calculates its Euclidean distance as shown in figure (5).

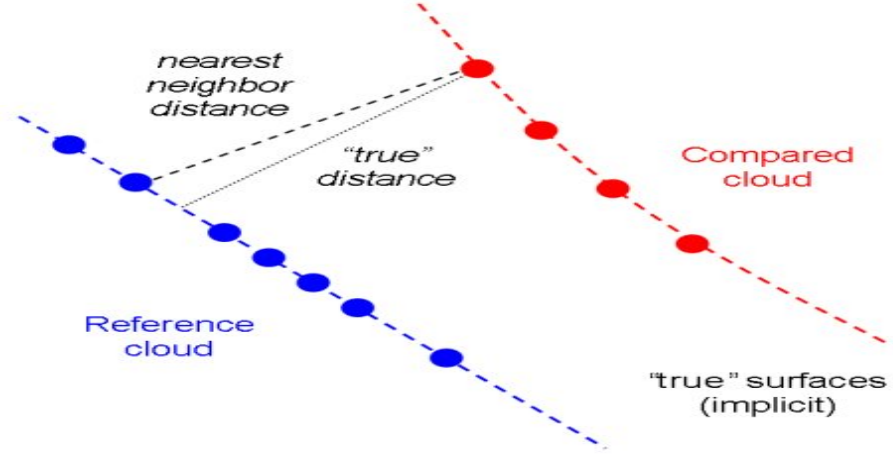

Figure (5): The principle of the C2C distance method (Daniel G., 2015).

Improvements can be obtained by a local modeling option on $\mathrm{C} 2 \mathrm{C}$. There are three categories of local models based on the least-square best-fitting plane that goes through the nearest point and its neighbors: Least squares plane, 2D1/2 triangulation, and Quadric (formerly called 'Height function'). The quadratic function is unique for smooth surfaces. It is better to use the quadratic model due to its versatility. In fact the corresponding model is a quadratic function (6 parameters: $\left.Z=a \cdot X^{2}+b \cdot X+c \cdot X Y+d . Y+e . Y^{2}+f\right)$. In our case, a local height function is computed using the neighboring points within a radius of $10 \mathrm{~m}$ of the closest point in the reference cloud. As shown in figure (6), the measured distance is sensitive to the cloud's roughness, outliers and point spacing (Daniel G., 2015). 


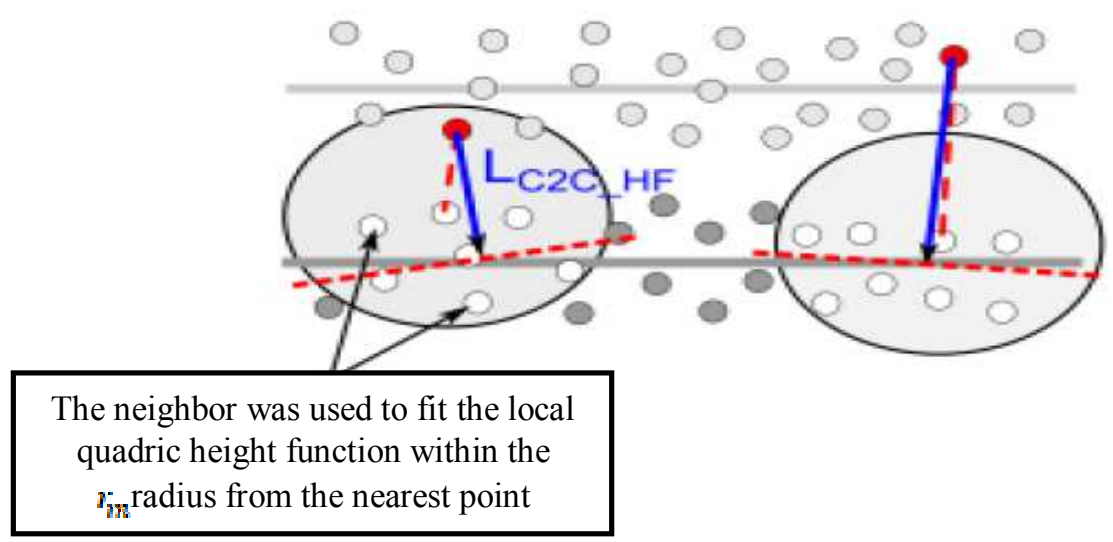

Figure (6): Closest point with local height function $L_{\mathrm{c} 2 c-H F}($ Daniel G., 2015).

\section{RESULTS AND DISCUSSION}

Figure (7,8 and 9) shows the point clouds raw multispectral LiDAR data of the study area and the DTMs of three channels that resulting from using ENVI -LIDAR software with a TIN filtering algorithm in additional to DTMs that generated from ATIN and ETEW filtering algorithms by ALDPAT software. The results show that the DTMs generated from channel-1 and 2 are very close due to the elevation and intensity values between channels- 1 and 2 are very close as shown in figure (3). Because there is no significant difference between the filtered and reference surfaces, the quantitative analysis will be carried out by using cloud compare software. In particular, the steps of visualization, and analysis of DTMs will be done using Global Mapper 16 software.

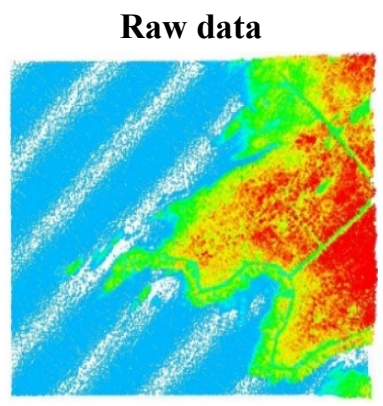

ENVI-TIN

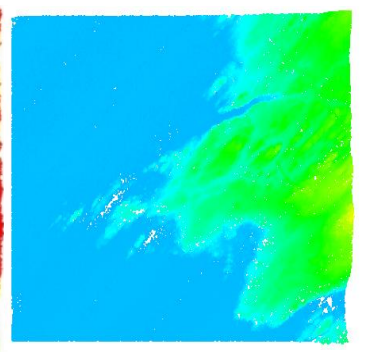

Figure (7): Point clouds raw data and filtered

Raw data

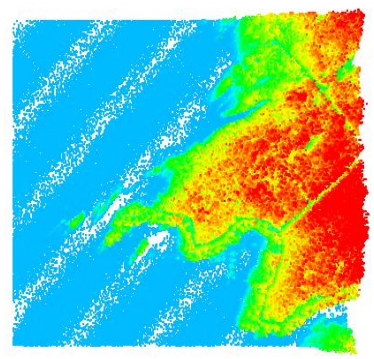

ENVI-TIN

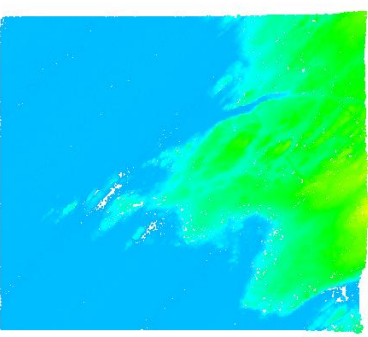

ATIN

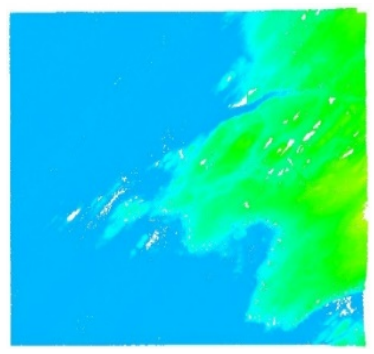
for channel-1.

ATIN

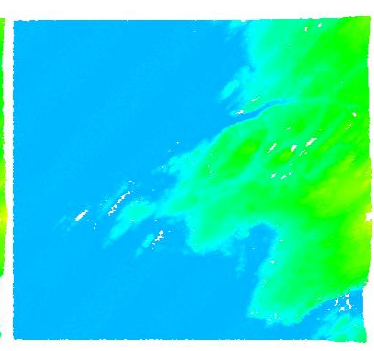

ETEW
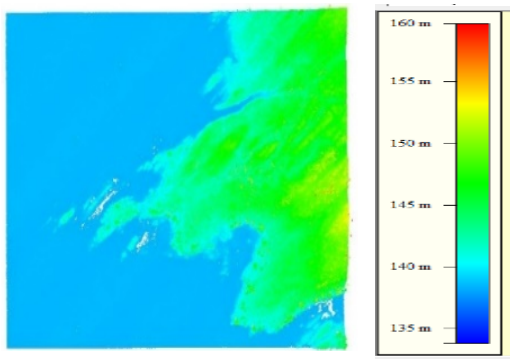

Figure (8): Point clouds raw data and filtered point clouds for test area by ENVI-TIN, ATIN, and ETEW for channel-2. 
Raw data

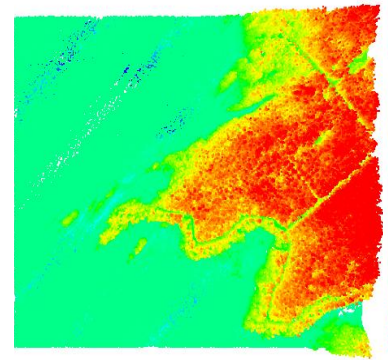

ENVI-TIN

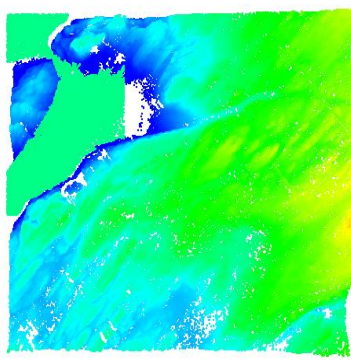

ATIN

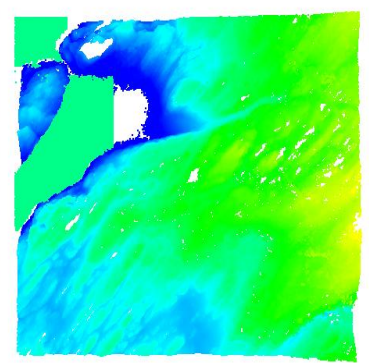

ETEW

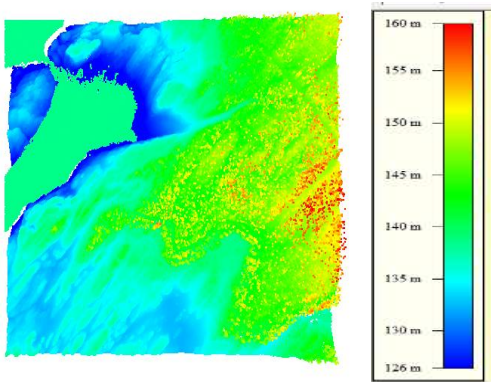

Figure (9): Point clouds raw data and filtered point clouds for test area by ENVI-TIN, ATIN, and ETEW for channel-3.

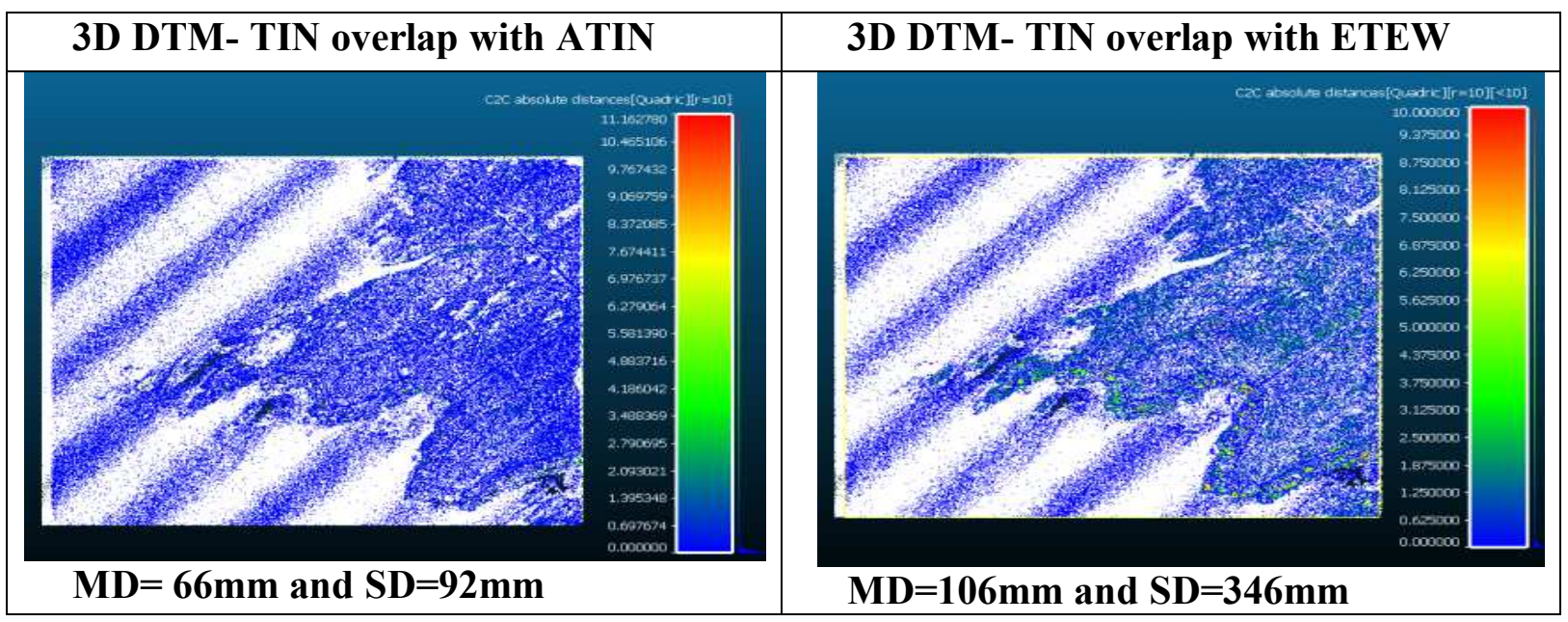

Figure (10): Results about comparing data on cloud compare channel-1.

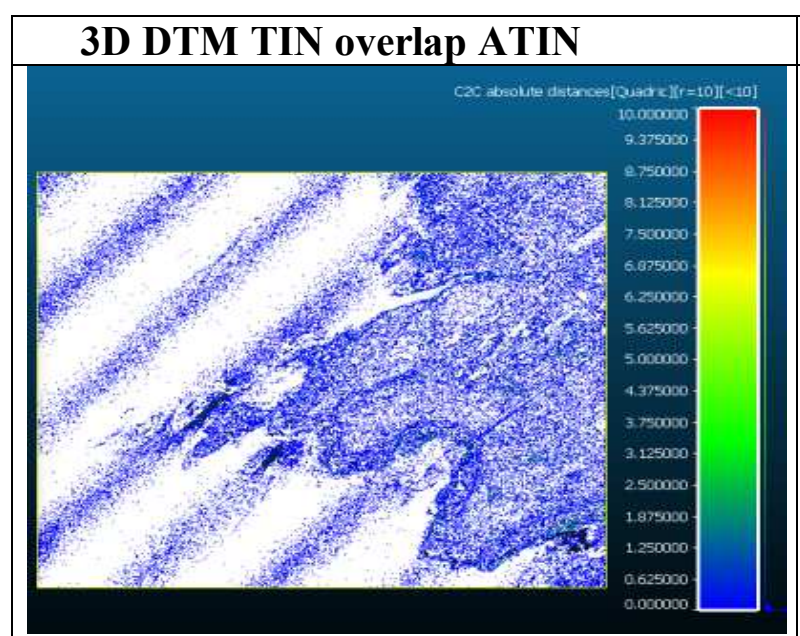

$\mathrm{MD}=66 \mathrm{~mm}$ and $\mathrm{SD}=97 \mathrm{~mm}$

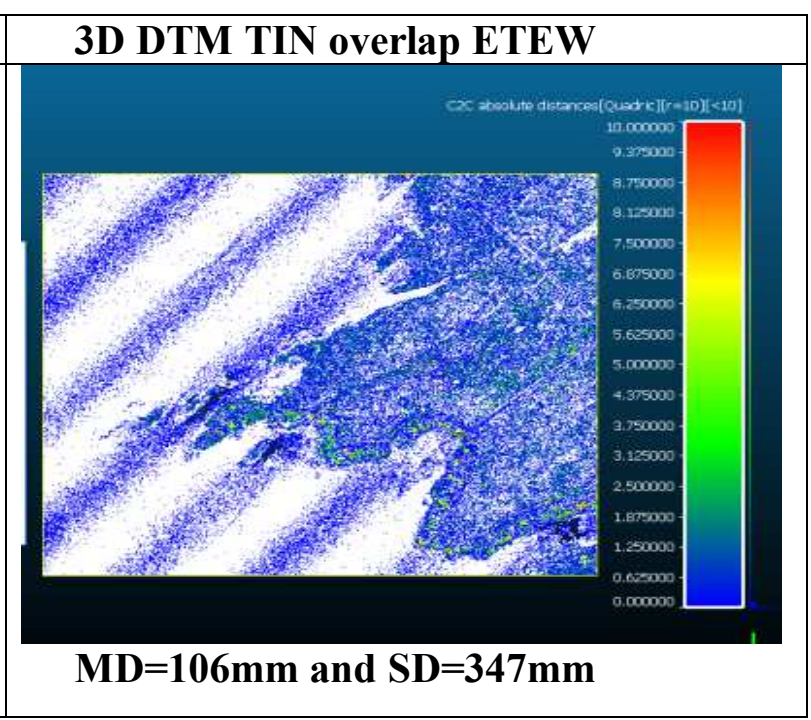

$\mathrm{MD}=106 \mathrm{~mm}$ and $\mathrm{SD}=347 \mathrm{~mm}$

Figure (11): Results about comparing data on cloud compare channel-2. 


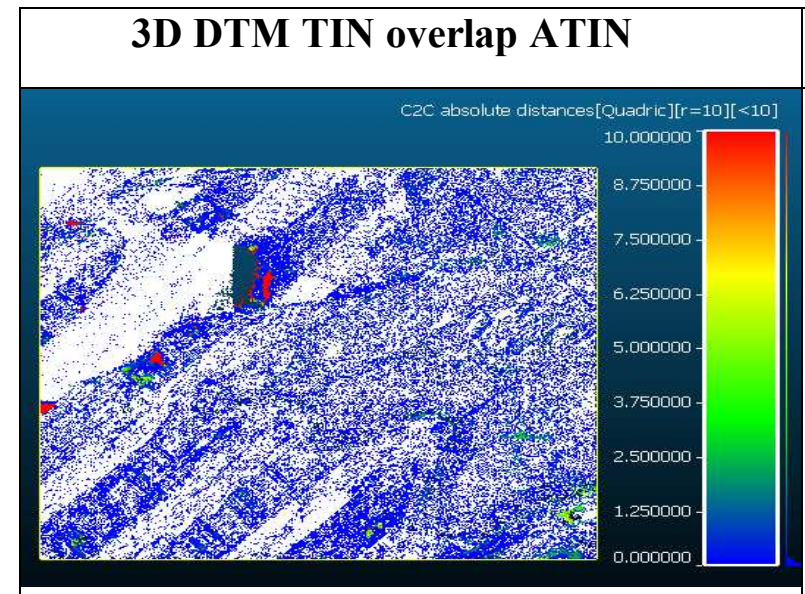

$\mathrm{MD}=145 \mathrm{~mm}$ and $\mathrm{SD}=561 \mathrm{~mm}$

\section{D DTM TIN overlap ETEW}

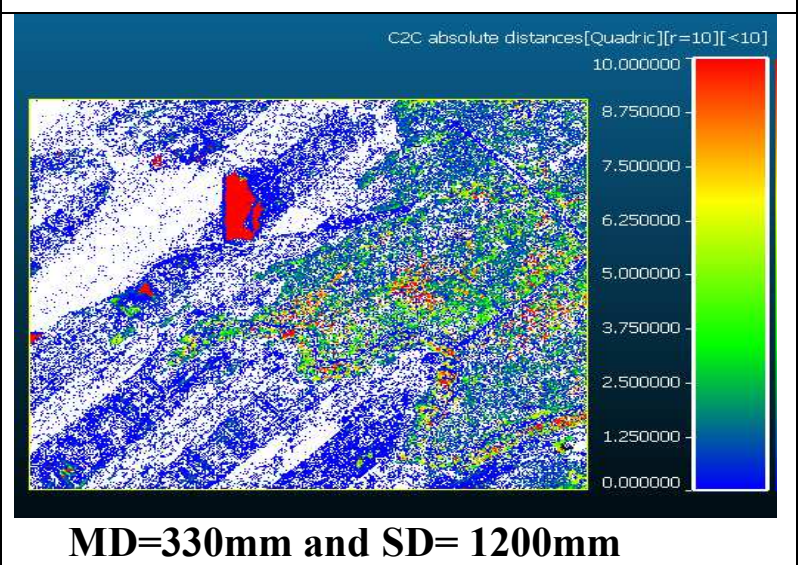

$\mathrm{MD}=330 \mathrm{~mm}$ and $\mathrm{SD}=1200 \mathrm{~mm}$

Figure (12): Results about comparing data on cloud compare channel-3.

\subsection{Results about comparing data on cloud compare for three channels.}

The Cloud to Cloud (C2C) absolute distance method using cloud compare software was using the two filters for the three channels. The six output DTMs produced were compared with DTMs produced by its corresponding ENVI-LiDAR and the results are shown in figure $(8,9,10)$. The blue color in the diagram means the data is nearest to the DTM created by ENVI-LiDAR while the red color shows that the deviation is in the maximum state. In the ETEW C2C channel-3 result, green color appears at certain spots. These green colors mean that the points have a standard deviation higher than ATIN. However, there is a similarity in the deviation created by the ATIN filter. Also, this software provides extra information to ease users in the decision-making process.

As shown in figure $(10,11$ and 12) two values can be calculated: mean distance and standard deviation. These statistical values for each channel are represented separately in three graphs as shown in figure (13). From the results, channel-1 standard deviation for ATIN is $0.092 \mathrm{~m}$ while for ETEW filter standard deviation is $0.346 \mathrm{~m}$. As for channel-2 ATIN and ETEW filter, the standard deviation values are 0.097 and $0.347 \mathrm{~m}$ respectively. According to channel3 ATIN and ETEW filter, standard deviation values are 0.561 and $0.579 \mathrm{~m}$.

From the results presented, the ATIN filter has demonstrated its efficacy in the three different channels compared with the performance of the ETEW for the used study area that consisting mainly of densely forested areas with the terrain and coastal area.

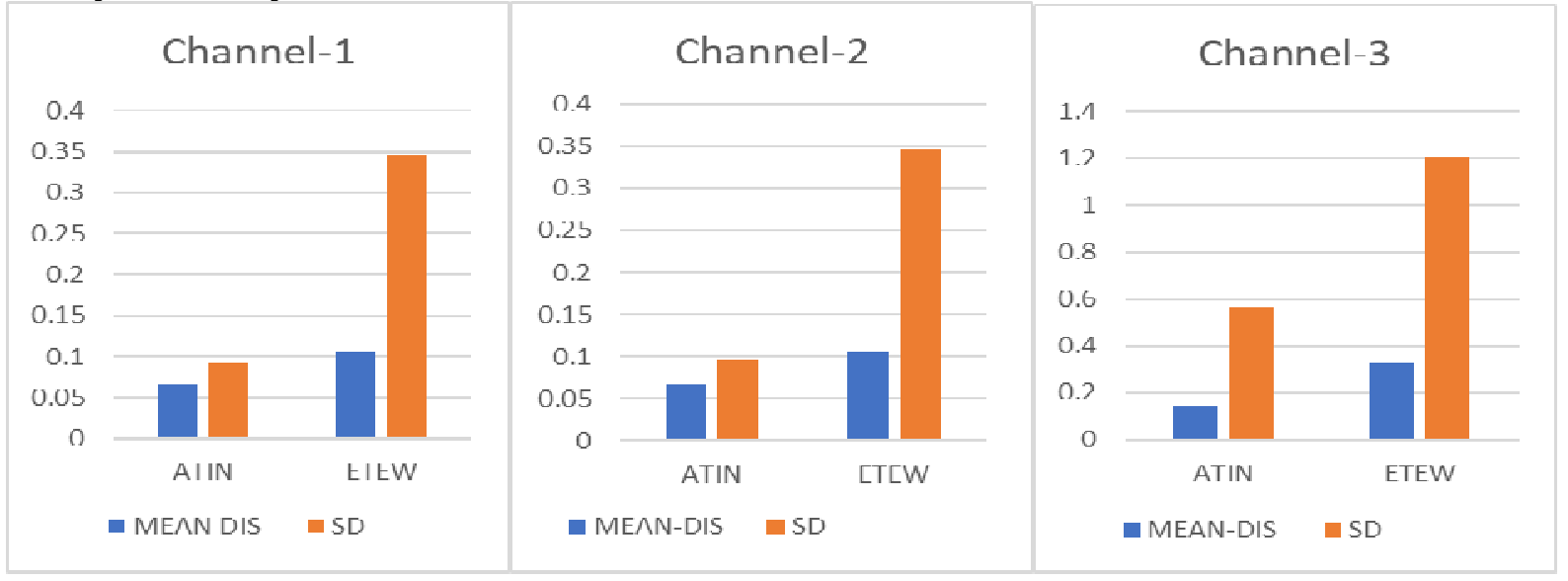

Figure (13): C2C Results about comparing data on cloud compare for three channels. 


\section{CONCLUSIONS}

Multi-spectral LiDAR point clouds provide more information about the terrain surface than mono-wavelength LiDAR, especially in water bodies' areas. The TITAN LiDAR provided by Teledyne Optech is an example of the multispectral LiDAR system. It has three different wavelengths: mid-infrared $(\mathrm{CH}-1)$, near-infrared $(\mathrm{CH}-2)$, and green $(\mathrm{CH}-3)(1.550,1.064$, and $0.532 \mu \mathrm{m})$ respectively. This study aims to compare the performance of two open-source filtering algorithms are adaptive TIN (ATIN) and Elevation Threshold with Expansion Window (ETEW) in the study area consist mainly of a water surface, coastal islands, and terrain area covered with dense plants and trees. To meet this objective, free sample multispectral LiDAR data covers a natural coastal area located at the Southwest tip of Tobermory (Ontario, Canada), which was kindly provided by the ISPRS Commission III on 'Remote Sensing'-and WG 111/5on 'Information Extraction from LiDAR Intensity Data'.

The study focused on assessing the performance of both ATIN and ETEW ground filtering algorithms in quantitative terms, using Cloud to Cloud (C2C) absolute distance method by cloud compares software that has the ability to directly compare three-dimensional between point clouds converting point clouds to grids or meshing data. The results showed that the efficiency of the ATIN filter performance to give DTMs are close to that generated from ENVI-LiDAR, with standard deviation values of \pm 92 and $\pm 97 \mathrm{~mm}$ for channel- 1 and channel -2 respectively. While in case of channel-3 where the terrain underwater appeared due to the ability of green wavelength to penetrate water surface, the performance of ATIN also give result better than ETEW, but the standard deviation value of channel -3 is higher than values of the standard deviations of channel -1 and 2, as a result of the appearance of terrain in the water area and the appearance of some sudden changes in height.

Acknowledgments: The authors would like to thank Dr. Ahmed Shaker the Vice-President of ISPRS - Technical Commission III and Dr. Marco Scaioni Chair of ISPRS WG III/5: Information Extraction from LiDAR Intensity Data for kindly providing multispectral airborne LiDAR data.

\section{REFERENCES}

1. Alganci U., Besol B. and Sertel E., (2018)."Accuracy Assessment of Different Digital Surface Models." ISPRS International Journal of Geo-Information, 7(3), pp.114.

2. Axelsson P., (2000). "DEM Generation from Laser Scanner Data Using Adaptive TIN Models." Int. Arch. of Photogram. and Remote Sens., B4, vol. 33, pp. 110-117.

3. Badenko V., Zotov D., Muromtseva N., Volkova Y. and Chernov P., (2019). "Comparison of Software for Airborne Laser Scanning Data Processing in Smart City Applications." ISPRS - International Archives of the Photogrammetry, Remote Sensing and Spatial Information Sciences XLII-5/W2 (September): pp.9-13. https://doi.org/10.5194/isprs-archives-xlii-5-w2-9-2019.

4. Chen Z.. (2018). "Convolutional Neural Networks for Land-cover Classification Using Multispectral Airborne Laser Scanning Data." (M.SC., University of Waterloo).

5. Dai W., Yang B., Dong Z. and Shaker, A., (2018). "A New Method for 3D Individual Tree Extraction Using Multispectral Airborne LiDAR Point Clouds." ISPRS journal of photogrammetry and remote sensing, 144, pp.400-411.

6. Daniel G., (2015). "Cloud Compare 3D Point Cloud and Mesh Processing Software." Webpage: Http://Www.Cloudcompare.Org.

7. Harris Corporation, (2019). Website, L3 Harris Geospatial Documentation Center, https://www.harrisgeospatial.com/docs/using_envi_lidar_Home.html.

8. Lague D., Brodu N. and Leroux J., (2013)."Accurate 3D Comparison of Complex Topography with Terrestrial Laser Scanner: Application to the Rangitikei Canyon (NZ)". ISPRS journal of photogrammetry and remote sensing, 82, pp.10-26.

9. Ma L., Chen Z., Li Y., Zhang D., Li J. and Chapman M.A., (2019). "Multispectral Airborne Laser Scanning Point-Clouds for Land Cover Classification Using Convolutional Neural Networks." International Archives of the Photogrammetry, Remote Sensing \& Spatial Information Sciences.

10. Matikainen L., Karila K., Hyyppa J., Litkey P., Puttonen E. and Ahokas E., (2017). "Object-Based Analysis of Multispectral Airborne Laser Scanner Data for Land Cover 
Classification and Map Updating." ISPRS Journal of Photogrammetry and Remote Sensing, 128, pp.298-313.

11. Morsy S., Shaker A. and El-Rabbany A., (2018)."Using Multispectral Airborne LiDAR Data for Land/Water Discrimination: A Case Study at Lake Ontario, Canada." Applied Sciences, 8(3),pp.349.

12. Morsy S., Shaker A. and El-Rabbany A., (2017), May. "Evaluation of Distinctive Features for Land/Water Classification from Multispectral Airborne LiDAR Data at Lake Ontario." In Proceedings of the 10th International Conference on Mobile Mapping Technology (MMT) (pp. 6-8).

13. Serifoglu C., Gungor O. and Yilmaz V., (2016)."Performance Evaluation of Different Ground Filtering Algorithm for UAV-Based Point Clouds." International Archives of the Photogrammetry, Remote Sensing \& Spatial Information Sciences, 41.

14. Shaker A., Yan W.Y. and LaRocque P.E., (2019). "Automatic Land-water Classification Using Multispectral Airborne LiDAR Data for Near-Shore and River Environments." ISPRS Journal of Photogrammetry and Remote Sensing, 152, pp.94108.

15. Sulaiman N.S., Maiid Z. and Setan H., (2010)."DTM Generation from LiDAR Data by Using Different Filters in Open-Source Software". Geoinformation Science Journal, 10(2), pp.89-109.

16. Suleymanoglu B. and Soycan M., (2019)."Comparison of Filtering Algorithms Used for DTM Production from Airborne LiDAR Data: A Case Study in Bergama, Turkey." Geodetski Vestnik, 63(3), pp.395-413.

17. Optech T., (2015). 'Multispectral LiDAR system: High Precision Environmental Mapping, Retrieved from http://www.TeledyneOptech.com/WpContent/Uploads/Titan- Specsheet-150515-WEB.pdf.

18. Vosselman G., (2000). "Slope Based Filtering of Laser Altimetry Data." International Archives of Photogrammetry and Remote Sensing, 33, pp.935-942.

19. Zhang K., Chen S.C., Whitman D., Shyu M.L., Yan J., Zhang C., (2003). "A Progressive Morphological Filter for Removing Nonground Measurements from Airborne LIDAR Data." IEEE Transactions on Geoscience and Remote Sensing, 41(4), pp. 872-882. doi:https://doi.org/10.1109/TGRS.2003.810682.

20. Zhang, K. and Cui Z., (2007). Airborne LIDAR Data Processing and Analysis Tools ALDPAT 1.0.WWWdocument, http://lidar.ihrc.fiu.edu/download/Doc/ALDPAT.pd

21. Zhang, K., and Whitman, D., (2005). "Comparison of Three Algorithms for Filtering Airborne Lidar Data." Photogrammetric Engineering \& Remote Sensing, 71(3), pp.313-324. 